\title{
On Fibonacci-type polynomial recurrences of order two and the accumulation points of their set of zeros
}

\author{
Prashant Batra \\ Hamburg University of Technology, Inst. for Reliable Computing, D-21071 Hamburg \\ batra@tuhh.de
}

Submitted December 18, 2017 - Accepted May 28, 2018

\begin{abstract}
We identify the accumulation points of the zero set of the polynomial family $G_{n+1}(z):=z G_{n}(z)+G_{n-1}(z), n \in \mathbb{N}$, generated from complex polynomial seeds $G_{0}, G_{1}$. This problem has been treated recently, for seed pairings of constants with linear polynomials, by Böttcher and Kittaneh (2016). We determine the accumulation points in the general case of arbitrary co-prime polynomial seeds, thus simplifying and streamlining previous approaches.
\end{abstract}

Keywords: Fibonacci polynomials; three-term recurrences; zero attractor; asymptotic zero location.

MSC: Primary: 11B39. Secondary: 30C15; 30B15; 40A15.

\section{Introduction}

The Fibonacci recursion $\varphi_{n+1}:=\varphi_{n}+\varphi_{n-1}, n \in \mathbb{N}$, with initial values $\varphi_{0} \equiv 0$, $\varphi_{1} \equiv 1$, can be generalized to complex polynomials, for fixed given $G_{0}, G_{1} \in \mathbb{C}[z]$, as

$$
G_{n+1}\left(G_{0}, G_{1} ; z\right) \hat{=} G_{n+1}(z):=z G_{n}(z)+G_{n-1}(z), \quad n \in \mathbb{N} .
$$

For $G_{0} \equiv 0, G_{1} \equiv 1$, we obtain the well-known Fibonacci polynomials which we denote by $F_{n}(z):=G_{n}(0,1 ; z)$. The roots of all the $F_{n}, n \in \mathbb{N}$, lie everywhere dense in $[-2 i, 2 i]$ (see [10]). For arbitrary co-prime polynomials $G_{0}, G_{1} \in \mathbb{C}[z]$ and $G_{n+1}$ 
defined by (1.1) we determine in the following the accumulation points arising from the set

$$
Z\left(G_{0}, G_{1}\right):=\left\{\xi \in \mathbb{C}: G_{n+1}(\xi)=0 \text { for at least one } n \in \mathbb{N}\right\}
$$

Let us denote the set-theoretic accumulation points of $Z\left(G_{0}, G_{1}\right)$ by $A\left(G_{0}, G_{1}\right)$. Mátyás [16] characterized the real accumulation points in $A\left(G_{0}, G_{1}\right)$ for general seed polynomials $G_{0}, G_{1} \in \mathbb{C}[z]$, and moreover determined them explicitly [14] for the real seeds $G_{0}:=-g, G_{1}(z):=z \pm g,(g \in \mathbb{R} \backslash\{0\})$. Recently, Böttcher and Kittaneh [5] determined all accumulation points for

$$
G_{0}(z):=a, \quad G_{1}(z):=z+b .
$$

They showed that for such seed pairings the accumulation points $A\left(G_{0}, G_{1}\right)$ contain $[-2 i, 2 i]$ together with at most two points, depending on the seeds.

The inclusion $[-2 i, 2 i] \subset A(a, z+b)$, as established in [5], relied on the identification (found in [15]) of $G_{n+1}(a, z+b ; z)$ as the characteristic polynomial of a perturbed tridiagonal Toeplitz matrix $T_{n+1}$, followed by an embedding of $T_{n+1}$ into an infinite Toeplitz matrix $T$, and an application of the finite section method in connection with $T$ 's essential spectrum.

In the following, we present our generalization and analysis. In Section 2 we determine, for arbitrary co-prime polynomial seeds $G_{0}, G_{1}$ the isolated points in $A\left(G_{0}, G_{1}\right)$ by a natural number-theoretic approach. This reveals moreover (see our Remark 2.3 below) the general meaning of the technical conditions in [5]. We avoid an obstacle to the direct generalization of the Böttcher-Kittaneh approach [5], namely, the missing general, computable Toeplitz matrix interpretation of the recurrence polynomials $G_{n+1}\left(G_{0}, G_{1} ; z\right)$.

In Section 3, looking at the elegant fixed point-argument in [5], we add the observation that the same argument essentially leads more generally to $[-2 i, 2 i] \subset$ $A\left(G_{0}, G_{1}\right)$. To this end, we rewrite the values of the polynomial $G_{m+1}$ at $x \in \mathbb{C}$ in terms of the solutions of the characteristic equation, and identify the general symmetric structure. Thus, different from [5], we avoid the discussion of the essential spectrum of operators and their truncations as well as convergence issues. Nevertheless, our proofs could be re-used in this direction. We close with some small historical notes in Section 4.

\section{The isolated accumulation points}

Let the Fibonacci polynomials be defined (as above) by

$$
F_{n+1}(z):=z F_{n}(z)+F_{n-1}(z), n \in \mathbb{N},
$$

where $F_{0}(z) \equiv 0, F_{1}(z) \equiv 1$, and hence $F_{2}(z)=z$. (Thus, every $F_{k}(z)$ is a polynomial of degree $k-1$, with $F_{k}(1)$ being a Fibonacci number.) It is wellknown, cf. [10], that the zeros of $F_{n+1}$ are the rotated, scaled zeros of the Chebyshev 
polynomials of the second kind of degree $n \in \mathbb{N}$. This implies in particular that no two consecutive Fibonacci polynomials have a common root. Let us note that if either $G_{0} \equiv 0$ or $G_{1} \equiv 0$, the polynomials $G_{n+1}$ will be the product of an $F_{k}$ by the non-trivial polynomial seed. Thus, we may omit these trivial cases from the discussion of the zero set and its accumulation points.

We first expand Theorem 1 in [16] characterizing zeros outside $[-2 i, 2 i]$.

Lemma 2.1. Assume that two polynomials $G_{0}, G_{1} \in \mathbb{C}[z] \backslash\{0\}$ are co-prime, i.e., let these have only trivial common divisors. Let us consider a value $x \in$ $Z\left(G_{0}, G_{1}\right) \backslash[-2 i, 2 i]$. Then for $G_{n+1} \in \mathbb{C}[z]$ defined by $G_{n+1}(z)=z G_{n}(z)+$ $G_{n-1}(z), n \in \mathbb{N}$, we have

$$
G_{n+1}(x)=0 \quad \Leftrightarrow \quad-\frac{G_{1}(x)}{G_{0}(x)}=\frac{F_{n-1}(x)}{F_{n}(x)} .
$$

Proof. As a generalization from Fibonacci numbers to Fibonacci polynomials it is easily proved by induction that

$$
\left(\begin{array}{cc}
F_{n+1}(z) & F_{n}(z) \\
F_{n}(z) & F_{n-1}(z)
\end{array}\right)=\left(\begin{array}{cc}
z & 1 \\
1 & 0
\end{array}\right)^{n}
$$

(this may be found, e.g., in [4]). Subsequently, matrix calculus establishes ( $c f$. , e.g., $[4,8])$ that

$$
G_{n+1}(z)=G_{1}(z) F_{n}(z)+G_{0}(z) F_{n-1}(z) \text { for } z \in \mathbb{C} .
$$

Hence, $G_{n+1}(x)=0$ is equivalent to $G_{1}(x) F_{n}(x)=-G_{0}(x) F_{n-1}(x)$. As the zeros of $F_{n}$ lie in $[-2 i, 2 i], c f .$, e.g. [10], we have $F_{n}(x) \neq 0$. As the polynomials $G_{0}$ and $G_{1}$ are co-prime, we see that $G_{0}(x) \neq 0$. Hence, $(2.1)$ holds true.

There is a natural analogue of the classical 'Binet formula' for the Fibonacci polynomials, and in view of (2.2), also for the polynomials $G_{n}$ (see, e.g., Mátyás [15]). To write out this generalization, we define $\lambda_{1}, \lambda_{2}$ by

$$
\begin{aligned}
& \lambda_{1}(z):=\frac{z}{2} \cdot\left(1+\sqrt{1+4 / z^{2}}\right), \\
& \lambda_{2}(z):=\frac{z}{2} \cdot\left(1-\sqrt{1+4 / z^{2}}\right) .
\end{aligned}
$$

Taking the principal value of the logarithm outside the purely imaginary interval $[-2 i, 2 i]=: J$ the $\lambda_{k}(\cdot)$ are analytic functions. Please note that in $\mathbb{C} \backslash J$ we have $\left|\lambda_{1}(z)\right|>\left|\lambda_{2}(z)\right|$. Thus, our choice of the $\lambda_{i}$ avoids the case distinctions found in [16]. Moreover, for any fixed $x \in \mathbb{C} \backslash J$ we have

$$
\begin{gathered}
\lambda_{1}(x)+\lambda_{2}(x)=x, \quad \text { and } \\
\lambda_{1}(x) \cdot \lambda_{2}(x)=-1 .
\end{gathered}
$$


Hence, $z^{2}-x \cdot z-1=\left(z-\lambda_{1}(x)\right) \cdot\left(z-\lambda_{2}(x)\right)$.

With these definitions, substituting $x \notin\{-2 i, 0,2 i\}$ into (2.2), the evaluation of $G_{n+1}$ at $x$ can be rewritten as

$$
G_{n+1}(x)=G_{1}(x) \frac{\lambda_{1}^{n+1}(x)-\lambda_{2}^{n+1}(x)}{\lambda_{1}(x)-\lambda_{2}(x)}+G_{0}(x) \frac{\lambda_{1}^{n}(x)-\lambda_{2}^{n}(x)}{\lambda_{1}(x)-\lambda_{2}(x)} .
$$

As in [16], and similar to, e.g., [13, 7], we express the values of the Fibonacci-like polynomials $G_{n}$ generated by the recurrence as

$$
G_{n}(x)=w_{1}(x) \cdot \lambda_{1}^{n}(x)-w_{2}(x) \cdot \lambda_{2}^{n}(x) \quad \text { for } \quad x \in \mathbb{C} \backslash\{-2 i, 2 i, 0\},
$$

with

$$
w_{1}(x):=\frac{G_{1}(x)-\lambda_{2}(x) \cdot G_{0}(x)}{\lambda_{1}(x)-\lambda_{2}(x)}, \quad \text { and } \quad w_{2}(x):=\frac{G_{1}(x)-\lambda_{1}(x) \cdot G_{0}(x)}{\lambda_{1}(x)-\lambda_{2}(x)} .
$$

The generalization of the continued fraction expansion for the (inverse of the) golden ratio, i.e., the fact that

$$
\lim _{n \rightarrow \infty} \frac{F_{n-1}(1 / x)}{F_{n}(1 / x)}=\frac{-1+\sqrt{1+4 x^{2}}}{2 x}
$$

inside the doubly-slit complex plane $\mathbb{C} \backslash((-\infty,-i / 2] \cup[i / 2,+\infty)])$ is well-known (cf., e.g., [9]). This easily leads us to the determination of the points in $A\left(G_{0}, G_{1}\right)$ which lie outside $[-2 i, 2 i]$.

Theorem 2.2. Given co-prime polynomials $G_{0}, G_{1} \in \mathbb{C}[z] \backslash\{0\}$. A complex value $x^{\prime} \in \mathbb{C} \backslash[-2 i, 2 i]$ is an accumulation point of the zero set $Z\left(G_{0}, G_{1}\right)$ if and only if

$$
\frac{G_{1}\left(x^{\prime}\right)}{G_{0}\left(x^{\prime}\right)}=\frac{x^{\prime}}{2} \cdot\left(1-\sqrt{1+4 / x^{\prime 2}}\right)=\lambda_{2}\left(x^{\prime}\right) .
$$

Proof. Relying on Lemma 2.1, we deduce from (2.1) together with (2.7) (or the elementary (2.5), employing the inequality $\left|\lambda_{2}\left(x^{\prime}\right) / \lambda_{1}\left(x^{\prime}\right)\right|<1$ ) for accumulation points $x^{\prime} \in Z\left(G_{0}, G_{1}\right) \cap(\mathbb{C} \backslash[-2 i, 2 i])$ existence of an infinite sequence of indices $n_{k}$ with

$$
x_{n_{k}} \quad \rightarrow \quad x^{\prime} \quad \text { and } \quad-\frac{G_{1}\left(x_{n_{k}}\right)}{G_{0}\left(x_{n_{k}}\right)} \rightarrow-\frac{G_{1}\left(x^{\prime}\right)}{G_{0}\left(x^{\prime}\right)}=\frac{-1+\sqrt{1+4\left(1 / x^{\prime}\right)^{2}}}{2 / x^{\prime}} .
$$

Hence,

$$
\frac{G_{1}\left(x^{\prime}\right)}{G_{0}\left(x^{\prime}\right)}=\frac{-x^{\prime}}{2}\left(-1+\sqrt{1+4 /\left(x^{\prime}\right)^{2}}\right)=\lambda_{2}\left(x^{\prime}\right) .
$$

Thus, an accumulation point outside $[-2 i, 2 i]$ is necessarily a zero of the cofactor $w_{1}(\cdot)$ in (2.6). We extract from [3] the essentials (fitting our tailored set-up) to show sufficiency of this condition. 
Choose a small circular neighbourhood of $x^{\prime}$ non-intersecting with $[-2 i, 2 i]$, say $D_{\epsilon}\left(x^{\prime}\right):=\left\{z \in \mathbb{C}:\left|z-x^{\prime}\right|<\epsilon\right\}$, such that its boundary $\partial D_{\epsilon}=\left\{z \in \mathbb{C}:\left|z-x^{\prime}\right|=\epsilon\right\}$ contains no zero of $w_{1}$. On the disc and its boundary, we consider

$$
w(z):=-w_{2}(z) \cdot \lambda_{2}^{n}(z) / \lambda_{1}^{n}(z) .
$$

On $\partial D_{\epsilon}$ we have $\left|w_{1}(z)\right|>m>0$, and $\left|\lambda_{2}(z) / \lambda_{1}(z)\right|<r<1$, for some constants $r$ and $m$.

Let $M:=\max _{z \in \partial D_{\epsilon}}\left\{\left|w_{1}(z)\right| ;\left|w_{2}(z)\right|\right\}$. Choose $N \in \mathbb{N}$ such that $2 M r^{N}<m$. Thus, for all $n \geq N$ we have $|w(z)|<\left|w_{1}(z)\right|$. Hence by Rouché's theorem ( $c f$., e.g., [1, p.153]), the two functions $w_{1}(z)-w(z)$ and $w_{1}(z)$ have the same number of zeros in $D_{\epsilon}$. Thus, as $x^{\prime} \in D_{\epsilon}$, and $w_{1}\left(x^{\prime}\right)=0$, there is at least one point $y_{n}$ in $D_{\epsilon}$ such that $w\left(y_{n}\right)=w_{1}\left(y_{n}\right)$, and hence $G_{n}\left(y_{n}\right)=0$ for all $n \geq N$.

Remark 2.3. The accumulation points $x^{\prime}$ outside $[-2 i, 2 i]$ may be found from $(2.3)$ and (2.4) via

$$
x^{\prime}=\lambda_{2}\left(x^{\prime}\right)+\lambda_{1}\left(x^{\prime}\right)=\lambda_{2}\left(x^{\prime}\right)-\frac{1}{\lambda_{2}\left(x^{\prime}\right)}=\frac{G_{1}\left(x^{\prime}\right)}{G_{0}\left(x^{\prime}\right)}-\frac{G_{0}\left(x^{\prime}\right)}{G_{1}\left(x^{\prime}\right)}
$$

as solutions of a polynomial equation in $x^{\prime}$. Of course, only those solutions $x^{\prime}$ with $\Re \frac{G_{1}\left(x^{\prime}\right)}{G_{0}\left(x^{\prime}\right)}<1$ can satisfy $(2.8)$.

\section{The segment of accumulation points}

It remains to determine the accumulation points in $[-2 i, 2 i]$.

Theorem 3.1. Consider two co-prime polynomials $G_{0}, G_{1} \in \mathbb{C}[z] \backslash\{0\}$ and the polynomial family $G_{n+1}, n \in \mathbb{N}$, defined by (1.1). Then every point $x^{\prime}$ in the imaginary segment $[-2 i, 2 i]$ is an accumulation point of the zero set $Z\left(G_{0}, G_{1}\right)$, i.e., we have $[-2 i, 2 i] \subset A\left(G_{0}, G_{1}\right)$.

Proof. We will show that all values $x^{\prime}$ in a dense subset of the disjoint open intervals $(-2 i, 0)$ and $(0,2 i)$ are accumulation points of $Z\left(G_{0}, G_{1}\right)$. This suffices to establish that $[-2 i, 2 i] \subset A\left(G_{0}, G_{1}\right)$. Let us transform the algebraic relation $G_{m+1}(x)=0, m \in \mathbb{N}$, into a two-variable equation with related fixed point problem. Using (2.5), we multiply $G_{m+1}(x)=0$ to obtain $\left(\lambda_{1}(x)-\lambda_{2}(x)\right) G_{m+1}(x)=0$ $\Leftrightarrow G_{1}(x)\left(\lambda_{1}^{m+1}(x)-\lambda_{2}^{m+1}(x)\right)+G_{0}(x)\left(\lambda_{1}^{m}(x)-\lambda_{2}^{m}(x)\right)=0$. We replace $x$ by $\lambda_{1}(x)+\lambda_{2}(x)=\lambda_{1}(x)-1 / \lambda_{1}(x)$ and find that

$$
\begin{gathered}
G_{1}\left(\lambda_{1}(x)-\frac{1}{\lambda_{1}(x)}\right)\left(\lambda_{1}^{m+1}(x)-\left(-\frac{1}{\lambda_{1}(x)}\right)^{m+1}\right)+ \\
G_{0}\left(\lambda_{1}(x)-\frac{1}{\lambda_{1}(x)}\right)\left(\lambda_{1}^{m}(x)-\left(-\frac{1}{\lambda_{1}(x)}\right)^{m}\right)=0 .
\end{gathered}
$$


This rational equation is of the form

$$
S\left(\lambda_{1}\right) \pm S\left(-1 / \lambda_{1}\right)=0, S \in \mathbb{C}[z] .
$$

The degree $\sigma$ of $S$ is bounded by $d+m+1$, where $d:=\max \left\{\operatorname{deg} G_{0} ; \operatorname{deg} G_{1}\right\}$.

Moreover, the least exponent of $\lambda_{1}$ in $S$ is at least $(m+1-d)$ for all sufficiently large $m$. Let us denote the reciprocal polynomial $(-z)^{\sigma} S(-1 / z)$ by $U(z)$. The exponents of $z$ in $U(z)$ thus lie in the range between 0 and $2 d$. We multiply the equation (3.1) by $\left(-\lambda_{1}\right)^{\sigma}$, incorporate signs appropriately, and obtain a polynomial equation of the form

$$
\lambda_{1}(x)^{2(m+1)} s\left(\lambda_{1}(x)\right)-U\left(\lambda_{1}(x)\right)=0,
$$

for some polynomials $s$ and $U \in \mathbb{C}[z]$ of degree at most $2 d$. The last equation may be rewritten and rearranged for $n:=m+1$, and $\varrho e^{i \theta}:=\lambda_{1}(x)$ (whenever $\left.U\left(\varrho e^{i \theta}\right) \neq 0\right)$ as

$$
\varrho^{2 n} e^{2 n i \theta}=\frac{s\left(\varrho e^{i \theta}\right)}{U\left(\varrho e^{i \theta}\right)}=: r(\varrho, \theta) e^{i \gamma(\varrho, \theta)} .
$$

This implicitly defines the functions $r$ and $\gamma$ depending on the variables $\varrho$ and $\theta$.

We demonstrate in the following the existence of values $x$ with $G_{m+1}(x)=0$ for all sufficiently large $m$ in any sufficiently small neighbourhood of $x^{\prime}=e^{i \varphi}-e^{-i \varphi} \in$ $(-2 i, 0) \cup(0,2 i)$ where $\varphi \in \mathbb{R}$ and $U\left(e^{i \varphi}\right) \neq 0$. This excludes the (finitely many) poles of modulus 1 eventually occuring in (3.2). Thus, at most finitely many, isolated points $x^{\prime}$ are excluded from $(-2 i, 0) \cup(0,2 i)$. The resulting point set is dense in $[-2 i, 2 i]$. The values $x$ are sought in the form $x=\varrho e^{i \theta}-\varrho^{-1} e^{-i \theta}$. Thus, for every sufficiently small $\epsilon, 0<\epsilon<1$, we define the parameter neighbourhood $X:=[1-\varepsilon, 1+\varepsilon] \times[\varphi-\varepsilon, \varphi+\varepsilon]$. If $\varepsilon>0$ is sufficiently small, the functions $r(\varrho, \theta)$ and $\gamma(\varrho, \theta)$ defined above in $(3.2)$ can be assumed to be continuously differentiable, and are bounded as, say, $0<\mu<r(\varrho, \theta)<M$ and $-M<\gamma(\varrho, \theta)<M$.

At this point, we may re-use the proof in [5] directly (without discussion of the essential spectrum or computation of perturbed Toeplitz determinants). For completeness, we repeat the nice and short argument based on fixed points.

There is an $n_{0}^{\prime} \in \mathbb{N}$ with the following property: for $n \geq n_{0}^{\prime}$ there is an integer $k_{n} \in \mathbb{Z}$ such that $\left|\pi k_{n} / n-\varphi\right|<\varepsilon / 2$. Since $e^{2 \pi i k_{n}}=1$, equation (3.2) is certainly satisfied if

$$
\varrho=[r(\varrho, \theta)]^{1 /(2 n)}, \quad \theta=\frac{1}{2 n} \gamma(\varrho, \theta)+\frac{\pi k_{n}}{n} .
$$

In other terms, equation (3.2) is satisfied if $(\varrho, \theta)$ is a solution of the fixed point equation $(\varrho, \theta)=F(\varrho, \theta)$ where

$$
F(\varrho, \theta):=\left([r(\varrho, \theta)]^{1 /(2 n)}, \frac{1}{2 n} \gamma(\varrho, \theta)+\frac{\pi k_{n}}{n}\right) .
$$

If $n$ is sufficiently large, then

$$
1-\varepsilon \leq \mu^{1 /(2 n)} \leq r(\varrho, \theta)^{1 /(2 n)} \leq M^{1 /(2 n)} \leq 1+\varepsilon,
$$


and

$$
\left|\frac{1}{2 n} \gamma(\varrho, \theta)+\frac{\pi k_{n}}{n}-\varphi\right| \leq \frac{1}{2 n} M+\frac{\varepsilon}{2} \leq \varepsilon .
$$

Consequently, $F$ maps $X$ into itself for every sufficiently large $n$. Denoting the partial derivatives as $\frac{\partial r}{\partial \varrho}=: r_{\varrho}, \frac{\partial r}{\partial \theta}=: r_{\theta}$ etc. the Jacobi matrix of $F$ reads

$$
\frac{1}{2 n}\left(\begin{array}{cc}
r^{1 /(2 n)-1} r_{\varrho} & r^{1 /(2 n)-1} r_{\theta} \\
\gamma_{\varrho} & \gamma_{\theta}
\end{array}\right) .
$$

The norm of this matrix goes to zero, uniformly in $(\varrho, \theta) \in X$, as $n$ goes to infinity. Thus, there is an $n_{0} \geq n_{0}^{\prime}$ such that $F$ is a strictly contractive map of $X$ into itself for $n \geq n_{0}$. Banach's fixed point theorem (see, e.g., [18]) therefore implies for each $n \geq n_{0}$ existence of a point $x=\varrho_{n} e^{i \theta_{n}}$ with $\left(\varrho_{n}, \theta_{n}\right) \in X=X(\epsilon)$ (with $\left.x^{\prime} \in X\right)$ such that $G_{n}(x)=0$. Letting $\epsilon \rightarrow 0$, we see that all the considered $x^{\prime}$ are accumulation points of the zero set. This carries over to the segment endpoints $-2 i, 2 i$ and the center 0 , as well as to the (eventually occurring finitely many) roots of $U(\cdot)$. Thus, the segment $[-2 i, 2 i]$ consists exclusively of accumulation points.

Future directions: The aim of this work was to give as simple and concise arguments as conceivable for the complete accumulation point determination of the considered recursions. Thereby, we wanted to re-connect to the elementary number-theoretic approach, while dealing with as many cases as possible. It would be interesting to see which higher-order recursions, or which recursions of the form $H_{n+1}(z)=p(z) H_{n}(z)+q(z) H_{n-1}(z)$, can be dealt with by elementary, concise arguments as the ones presented.

\section{Historical note}

The function $F_{n}(z)$ was considered in both the forms (2.5) and (2.6) as an arithmetical function of $n \in \mathbb{N}$ in the works of Lucas [13], Catalan [7], and, later, Bell [2]. Jacobsthal [11] considered the recursion $f_{n}(z):=f_{n-1}(z)+z f_{n-2}(z)$ (quite different from our (1.1)). A recent non-homogeneous generalization of this may be found in [12]. An early appearance of the Fibonacci polynomials $F_{n}(z)$ as a complex function of $z$ is in [6], see also $[4,10]$ and references therein. Sometimes the generalization we have considered here is called 'Fibonacci-like' as in [14, 15, 16], while the name 'Fibonacci-type' ( $c f$. ., e.g. $[8,5])$ seems to be more frequently used. The encompassing attribute 'generalized Fibonacci polynomials' is eventually used for solutions of other recurrences as well $c f$., e.g., [17].

\section{References}

[1] L.V. Ahlfors. Complex Analysis. McGraw-Hill, New York, N.Y., 3rd edition, 1979. 
[2] E.T. Bell. Arithmetical theorems on Lucas functions and Tchebycheff polynomials. American Journal of Mathematics, 57(4):781-788, 1935.

https://doi.org/10.2307/2371013

[3] S. Beraha, J. Kahane, and N.J. Weiss. Limits of zeros of recursively defined families of polynomials. In G.-C. Rota, editor, Studies in Foundations and Combinatorics, Adv. in Math. Suppl. Stud., 1, pages 213-232. Academic Press, New York-London, 1978 .

[4] M. Bicknell. A Primer for the Fibonacci Numbers: Part VII - An introduction to Fibonacci polynomials and their divisibility properties. Fibonacci Quarterly, 8(4):407420, 1970.

[5] A. Böttcher and F. Kittaneh. The limit of the zero set of polynomials of the Fibonacci type. Journal of Number Theory, 163:89-100, 2016.

https://doi.org/10.1016/j.jnt.2015.11.013

[6] P.F. Byrd. Expansion of analytic functions in polynomials associated with Fibonacci numbers. Fibonacci Quarterly, 1(1):16-29, 1963.

[7] E. Catalan. Note sur la théorie des fractions continues et sur certaines séries. Mémoires de l'académie impériale et royale des sciences et belles-lettres de Bruxelles, 45:3-82, 1883.

[8] M.X. He, P.E. Ricci, and D.S. Simon. Fibonacci-type polynomial as a trajectory of a discrete dynamical system. Rendiconti Del Circolo Matematico Di Palermo, Serie II, 51(2):367-374, 2002. https://doi.org/10.1007/bf02871661

[9] P. Henrici. Applied and Computational Complex Analysis, volume 2. John Wiley \& Sons, New York, N.Y., 1977.

[10] V.E. Hoggatt jr. and M. Bicknell. Roots of Fibonacci polynomials. Fibonacci Quart., 11(3):271-274, 1973.

[11] E. Jacobsthal. Fibonaccische Polynome und Kreisteilungsgleichungen. Sitzungsberichte Berliner Mathematische Gesellschaft, 17:43-51, 1918.

[12] C. Kimberling. Limits of polynomial sequences. Fibonacci Quarterly, 50(4):294-296, 2012 .

[13] E. Lucas. Théorie des fonctions numériques simplement périodiques. American Journal of Mathematics, 1(2):184-196, 1878. https://doi.org/10.2307/2369308

[14] F. Mátyás. The asymptotic behavior of the real roots of Fibonacci-like polynomials. Acta Acad. Paedagog. Agriensis, Sect. Math. (N.S.), 24:55-61, 1997.

[15] F. Mátyás. Bounds for the zeros of Fibonacci-like polynomials. Acta Acad. Paedagog. Agriensis, Sect. Math. (N.S.), 25:15-20, 1998.

[16] F. Mátyás. Real roots of Fibonacci-like polynomials. In K. Györy et al., (editors), Number Theory. Diophantine, computational and algebraic aspects. Proc. Int. Conference, Eger, Hungary, July 29 - August 2, 1996. deGruyter, Berlin, 1998. https://doi.org/10.1515/9783110809794.361

[17] A. Nalli and P. Haukkanen. On generalized Fibonacci and Lucas polynomials. Chaos, Solitons and Fractals, 42(5):3179-3186, 2009. https://doi.org/10.1016/j.chaos.2009.04.048 
[18] J. Stoer and R. Bulirsch. Introduction to Numerical Analysis. Springer-Verlag, New York, N.Y., 3rd edition, 2002.

https://doi.org/10.1007/978-0-387-21738-3 